\title{
Protective effect of CTRP6 on cerebral ischemia/reperfusion injury by attenuating inflammation, oxidative stress and apoptosis in PC12 cells
}

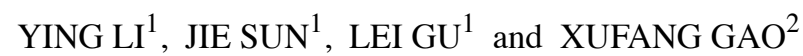 \\ ${ }^{1}$ Rehabilitation Centre, Beijing Xiaotangshan Hospital, Beijing 102211; \\ ${ }^{2}$ Department of Neurology, General Hospital of The Yangtze River Shipping and Wuhan Brain Hospital, \\ Wuhan, Hubei 430010, P.R. China
}

Received December 9, 2019; Accepted February 25, 2020

DOI: $10.3892 / \mathrm{mmr} .2020 .11108$

\begin{abstract}
The newly identified C1q/tumor necrosis factor (TNF)-related protein-6 (CTRP6) is a highly conserved paralog of adiponectin with modulatory effects on metabolism and inflammation. However, the role of CTRP6 in cerebral ischemia/reperfusion (I/R) injury remains unknown. The aim of the present study was to explore the protective effects of CTRP6 against cerebral I/R injury and elucidate the possible underlying mechanisms. Oxygen-glucose deprivation and reperfusion (OGD/R) was used to induce an I/R injury model in vitro. Western blotting, reverse transcription-quantitative PCR, ELISA and flow cytometry analysis were used to measure the levels of CTRP6 along with those of inflammation-, oxidative stress- and apoptosis-related cytokines. The results indicated that CTRP6 expression was markedly downregulated following $\mathrm{OGD} / \mathrm{R}$. OGD/R also increased i) the activities of pro-inflammatory factors TNF- $\alpha$, interleukin (IL)-1 $\beta$, IL-6 and the levels of the oxidative products reactive oxygen species and malondialdehyde; ii) the ratio of apoptotic PC12 cells and iii) the expression of the pro-apoptotic proteins Bax, cleaved caspase- 3 and cleaved caspase- 9 . In addition, the activities of the anti-inflammatory factors IL-10 and superoxide dismutase and the expression of the anti-apoptotic protein Bcl-2 were decreased. However, overexpression of CTRP6 rescued OGD/R-stimulated exacerbation of inflammation, oxidative stress and apoptosis. Mechanistically, OGD/R activated Ras homolog family member A (RhoA)/Rho-associated coiled-coil-containing protein kinase (Rock)/phosphatase and tensin homologue deleted on chromosome 10 (PTEN) signaling, whereas CTRP6 overexpression restored the
\end{abstract}

Correspondence to: Dr Xufang Gao, Department of Neurology, General Hospital of The Yangtze River Shipping and Wuhan Brain Hospital, 5 Huiji Road, Wuhan, Hubei 430010, P.R. China E-mail: gaoxufanglab@163.com

Key words: adiponectin, apoptosis, inflammation, oxidative stress, reperfusion injury expression of RhoA, Rock, PTEN, phosphoinositide 3-kinase (PI3K) and protein kinase B (Akt). Furthermore, when CTRP6 and RhoA were overexpressed at the same time, RhoA abolished the protective effects of CTRP6 overexpression on OGD/R-induced inflammation, oxidative stress and apoptosis, while the presence of a PTEN inhibitor recovered the protective effects of CTRP6. Taken together, the findings of the present study indicate that CTRP6 attenuates cerebral ischemia/reperfusion-induced inflammation, oxidative stress and apoptosis via inhibiting the RhoA/Rock/PTEN pathway, thereby activating PI3K/Akt signaling.

\section{Introduction}

Cerebral ischemia/reperfusion (I/R) injury, which coexists with ischemia over a certain time period, may result in serious cerebral microcirculatory damage and is a major cause of disability and mortality worldwide (1). Although considerable improvements in diagnosis, treatment and rehabilitation have been achieved (2), safe and effective therapeutic agents for cerebral I/R are still urgently needed (3).

Cerebral I/R injury involves multiple pathological processes, among which neuroinflammation, apoptosis and oxidative stress have been considered to play key roles in brain damage during cerebral I/R (4-6). Accumulating evidence has demonstrated the presence of excessive pro-inflammatory factors, pro-apoptotic proteins and oxidative stress products in cerebral I/R injury (6-8). Furthermore, previous studies indicated that the phosphoinositide 3-kinase (PI3K)/protein kinase B (Akt) pathway exerts neuroprotective effects via inhibiting inflammation, oxidative stress and apoptosis (9-11). Thus, targeting inflammation, oxidative stress and apoptosis may be a viable therapy option for cerebral I/R injury.

$\mathrm{C} 1 \mathrm{q} / \mathrm{tumor}$ necrosis factor (TNF)-related protein-6 (CTRP6) is a member of the adiponectin paralogs family of proteins designated as CTRPs. Although CTRPs share structural homology with adiponectin, each CTRP has a unique tissue distribution and exhibits diverse functions (12). CTRP6, which is expressed in adipose tissue, placenta, heart and brain, exerts modulating effects on energy metabolism and inflammation $(13,14)$. Considering that CTRP6 induces the expression 
of interleukin (IL)-10 via extracellular signal-regulated kinase $1 / 2$ activation in macrophages (15), it may represent a novel target for pharmacological therapy in inflammatory diseases. Of note, several studies have confirmed the protective effects of CTRP6 on inflammation-related diseases, such as myocardial fibrosis (16), cardiac injury (17) and arthritis (18), but the role of CTRP6 in I/R injury is rarely reported. Interestingly, as another member of the CTRP family, CTRP9 has been reported to protect against myocardial I/R injury via suppression of cell apoptosis $(19,20)$, but whether CTRP6 protects against cerebral $\mathrm{I} / \mathrm{R}$ injury remains elusive.

The small $G$ protein Ras homolog family member A (RhoA) and its downstream effector Rho-associated coiled-coil-containing protein kinase (Rock) extensively participate in the regulation of I/R injury in the myocardium, brain, liver or kidney (21). Previous evidence suggested that inhibition of RhoA/Rock signaling may initiate phosphatase and tensin homologue deleted on chromosome 10 (PTEN) activity to activate the PI3K/Akt pathway, which, in turn, prevents the inflammatory response in ischemic brain and liver models $(22,23)$. Moreover, CTRP6 was shown to improve cardiac function or attenuate post-infarct cardiac fibrosis via activation of Akt by targeting the RhoA pathway $(16,17)$.

Therefore, the present study was designed to i) determine the functions of CTRP6 in cerebral I/R injury; ii) investigate whether CTRP6 regulates cerebral I/R injury induced by oxygen-glucose deprivation and reperfusion (OGD/R) in PC12 cells and iii) determine whether RhoA or Akt are responsible for the actions of CTRP6 upon cerebral I/R injury, and elucidate the detailed mechanisms.

\section{Materials and methods}

Cell culture. PC12 cells (American Type Culture Collection) were cultured in standard growth medium (DMEM, Gibco; Thermo Fisher Scientific, Inc.), supplemented with 10\% FBS (Gibco; Thermo Fisher Scientific, Inc.) and $1 \%$ penicillin-streptomycin at $37^{\circ} \mathrm{C}$ in a $5 \% \mathrm{CO}_{2}$ atmosphere.

$O G D / R$. For induction of an in vitro $\mathrm{I} / \mathrm{R}$ injury model, $\mathrm{PC} 12$ cells were incubated in a deoxygenated glucose-free Hanks' Balanced Salt Solution within an anaerobic chamber $\left(5 \% \mathrm{CO}_{2}\right.$, $95 \% \mathrm{~N}_{2}$ ) at $37^{\circ} \mathrm{C}$ for $2 \mathrm{~h}$. After OGD treatment for $2 \mathrm{~h}$, the cells were removed from the anoxic atmosphere and transferred to a normal environment for $12 \mathrm{~h}$.

Plasmid constructs and cell transfection. Full-length cDNAs of rat CTRP6 or RhoA were cloned into the pcDNA3.1 vector (Thermo Fisher Scientific, Inc.). A pcDNA3.1 empty vector was used as a negative control. PC12 cells were transfected with $12.5 \mathrm{mg} / 1 \mathrm{pcDNA} 3.1-\mathrm{CTR}$ 6, pcDNA3.1-RhoA or pcDNA3.1 using Lipofectamine ${ }^{\circledR} 2000$ reagent (Invitrogen; Thermo Fisher Scientific, Inc.) according to the manufacturer's protocol. Following $48 \mathrm{~h}$ of transfection, the cells were collected for subsequent experiments.

Western blot analysis. To determine protein expression levels, total protein was extracted from PC12 cells using RIPA lysis buffer (Thermo Fisher Scientific, Inc.). All protein samples were quantified by a bicinchoninic acid kit (Thermo
Fisher Scientific, Inc) and equal amounts (50 $\mu \mathrm{g})$ of each sample were separated by $8-12 \%$ SDS-PAGE. Separated proteins were transferred onto PVDF membranes (Bio-Rad Laboratories, Inc.) and then blocked with 5\% non-fat milk at $37^{\circ} \mathrm{C}$ for $1 \mathrm{~h}$, after which time the membranes were incubated with the following primary antibodies (Abcam) overnight at $4^{\circ} \mathrm{C}$ : Anti-CTRP6 (cat. no. ab36900; 1:1,000), anti-Bcl2 (cat. no. ab32124; 1:1,000), anti-Bax (cat. no. ab32503; 1:1,000), anti-cleaved caspase-3 (cat. no. ab2302; 1:500), anti-caspase-3 (cat. no. ab13847; 1:500), anti-cleaved caspase-9 (cat. no. ab32539; 1:1,000), anti-caspase-9 (cat. no. ab2324; 1:400), anti-RhoA (cat. no. ab187027; 1:5,000), anti-Rock1 (cat. no. ab45171; 1:2,000), anti-Rock2 (cat. no. ab71598; 1:1,000), anti-PTEN (cat. no. ab32199; 1:10,000), anti-Akt (cat. no. ab8805; 1:500), anti-PI3K (cat. no. ab109006; 1:1,000), anti-phosphorylated-PI3K (cat. no. ab133595; 1:1,000), anti-phosphorylated-Akt (cat. no. ab81283; 1:5,000) and anti-GAPDH (Santa Cruz Biotechnology, Inc.; cat. no. Sc-47724; 1:1,000). Finally, the membranes were incubated with a horseradish-conjugated secondary antibody (goat anti-rabbit IgG; cat. no. ab205718; 1:10,000; Abcam) at room temperature for $2 \mathrm{~h}$. Protein bands were visualized using an electrochemiluminescence system (Amersham Imager 600; GE Healthcare) and an Immun-Star HRP chemiluminescent substrate kit (cat. no. 1705041; Bio-Rad Laboratories, Inc.) at room temperature for $2 \mathrm{~min}$. ImageJ version 1.8.0_112 software (National Institutes of Health) was used to quantify the expression levels of each protein.

$R N A$ isolation and reverse transcription-quantitative $P C R$ $(R T-q P C R)$ analysis. Total RNA was extracted from PC12 cells using TRIzol ${ }^{\circledR}$ reagent (Invitrogen; Thermo Fisher Scientific, Inc.), according to the manufacturer's protocol, and $1 \mu \mathrm{g}$ total RNA was converted to cDNA using the PrimeScript ${ }^{\mathrm{TM}}$ RT reagent kit with gDNA Eraser (Takara Biotechnology Co., Ltd.), according to the manufacturer's protocol. The following RT temperature protocol was used: $37^{\circ} \mathrm{C}$ for $15 \mathrm{~min}$ and $85^{\circ} \mathrm{C}$ for 5 sec. qPCR was performed using TB Green ${ }^{\circledR}$ Fast qPCR Mix (Takara Biotechnology Co., Ltd.), according to the manufacturer's protocol. Differential expression of mRNA was calculated using the $2^{-\Delta \Delta \mathrm{Cq}}$ method (24). The primers used were as follows: CTRP6, forward 5'-CCATCCTGAAAG GTGACAAAGG-3' and reverse 5'-AGTAATGCGTCTGGC ACGAG-3'; RhoA, forward 5'-CCAAAATGAAGCAGGAGC CG-3' and reverse 5'-ATGAGGCACCCCGACTTTTT-3'; and GAPDH, forward 5'-AGTGCCAGCCTCGTCTCATA-3' and reverse 5'-TGAACTTGCCGTGGGTAGAG-3'. The following thermocycling conditions were used for the qPCR: Initial denaturation at $95^{\circ} \mathrm{C}$ for $30 \mathrm{sec}$; and 40 cycles of $95^{\circ} \mathrm{C}$ for $5 \mathrm{sec}$ and $60^{\circ} \mathrm{C}$ for $15 \mathrm{sec}$; followed by a default melting curve.

MTT assay. The cell viability of PC12 cells under different conditions was assessed by MTT assay (Beyotime Institute of Biotechnology; cat. no. C0009), according to the manufacturer's protocol. In brief, cells that transfected with or without indicated plasmids were seeded in 96-well plates for $24 \mathrm{~h}$ and then incubated with $20 \mu \mathrm{l}$ MTT solution for $4 \mathrm{~h}$. After discarding MTT medium dissolving the remaining formazan crystals with DMSO, the cell viability was tested at $570 \mathrm{~nm}$ by a microplate reader (Thermo Fisher Scientific, Inc). 
Detection of inflammatory factors and oxidative stress levels. The levels of the pro-inflammatory cytokines TNF- $\alpha$ (cat. no. ab100785), IL-1 $\beta$ (cat. no. ab100768), IL-6 (cat. no. ab100772) and IL-10 (cat. no. ab100765) were detected in PC12 cells using their respective ELISA kits (Abcam), according to the manufacturer's protocol. A cellular reactive oxygen species (ROS; cat. no. ab113851) assay kit, superoxide dismutase (SOD; cat. no. ab65354) activity assay kit and lipid peroxidation product malondialdehyde (MDA; cat. no. ab118970) assay kit (Abcam) were used to determine ROS, SOD and MDA activity, respectively, according to the manufacturers' protocols.

Flow cytometry. Cell apoptosis was assessed by flow cytometry using propidium iodide (PI) staining. Briefly, cells were gently washed twice with PBS, digested with $0.25 \%$ trypsin and centrifuged at $200 \mathrm{x} \mathrm{g}$ at $4^{\circ} \mathrm{C}$ for $5 \mathrm{~min}$. After the resuspension of the cell pellet with $1 \mathrm{ml} \mathrm{NaCl} / \mathrm{Pi}$ supplemented with $100 \mu \mathrm{g} / \mathrm{ml}$ RNase, the cells were incubated with PI at $4^{\circ} \mathrm{C}$ for $15 \mathrm{~min}$ in the dark and immediately analyzed using a BD FACSCanto $^{\mathrm{TM}}$ II flow cytometer (BD Biosciences). Data were analyzed using FlowJo 7.6 software (FlowJo LLC).

Statistical analysis. All data are expressed as the mean \pm standard deviation and the statistical analyses were conducted using GraphPad Prism 6 (GraphPad Software, Inc.). Differences were calculated by Student's t-test or one-way ANOVA followed by Turkey's post hoc test for $\geq 3$ samples. $\mathrm{P}<0.05$ was considered to indicate statistically significant differences.

\section{Results}

CTRP6 expression is markedly downregulated after $O G D / R$. CTRP6 expression was first detected in PC12 cells subjected to OGD/R treatment and it was observed that CTRP6 protein expression was significantly reduced in OGD/R-treated cells (Fig. 1A and B). Further detection revealed that the CTRP6 mRNA level also decreased following OGD/R treatment (Fig. 1C). The alteration of CTRP6 expression suggested a role for CTRP6 in OGD/R-induced cerebral I/R injury in PC12 cells.

CTRP6 overexpression protects against OGD/R-induced inflammation, oxidative stress and cell apoptosis. To explore the specific role of CTRP6 in cerebral I/R injury, recombinant plasmids expressing CTRP6 were transfected into PC12 cells to overexpress CTRP6. As shown in Fig. 2A and B, CTRP6 protein and mRNA expression was significantly increased in cells treated with pcDNA3.1-CTRP6, suggesting the efficiency of CTRP6 overexpression.

To determine whether CTRP6 could alleviate OGD/R-induced inflammation, the activities of inflammatory molecules, including TNF- $\alpha$, IL-1 $\beta$, IL- 6 and IL-10, were detected. A significant increase in TNF- $\alpha$, IL- $1 \beta$ and IL- 6 and a decrease in the anti-inflammatory cytokine IL-10 were observed in OGD/R-treated cells (Fig. 3B-E), indicating the occurrence of inflammation induced by OGD/R. In addition, CTRP6 overexpression successfully rescued the activities of these inflammatory factors (Fig. 3B-E). These results suggested
A

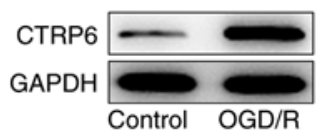

B

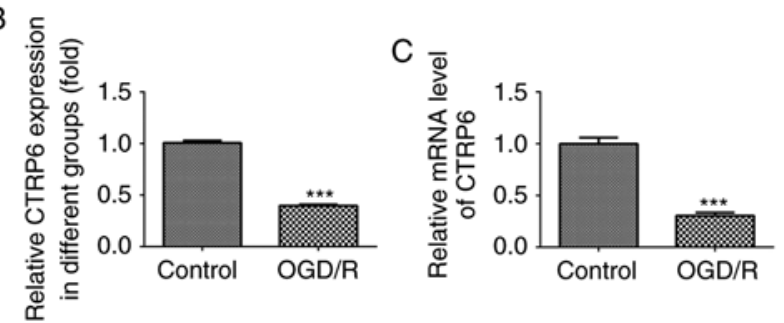

Figure 1. CTRP6 is downregulated after OGD/R. (A) Representative immunoblot analysis for CTRP6 in PC12 cells. (B) Relative CTRP6 protein expression normalized to the control $(n=3)$. (C) Alteration of the CTRP6 mRNA level ( $\mathrm{n}=3) .{ }^{* * *} \mathrm{P}<0.001$ vs. the control. CTRP6, C1q/tumor necrosis factor-related protein-6; OGD/R, oxygen-glucose deprivation and reperfusion.

that CTRP6 was able to inhibit OGD/R-induced inflammation in $\mathrm{PC} 12$ cells.

Oxidative stress also plays a key role in cerebral I/R injury (4) and the present results demonstrated that the levels of the oxidative products ROS and MDA were upregulated following OGD/R treatment (Fig. 3F and 3G), while the level of the antioxidant enzyme SOD was significantly reduced (Fig. 3H). Of note, the levels of ROS, MDA and SOD were also partially restored by CTRP6 overexpression (Fig. 3F-H).

The OGD/R-induced apoptosis was significantly rescued by CTRP6 overexpression. As shown in Fig. 4A, CTRP6 reduced the ratio of apoptotic cells. However, the expression of apoptosis-related proteins, including Bcl-2, Bax, cleaved caspase- 3 and caspase- 9 , was partially recovered when OGD/R treatment was given in combination with CTRP6 overexpression (Fig. 4B-F). Taken together, these findings indicate that CTRP6 may alleviate cerebral I/R injury via inhibiting inflammation, oxidative stress and apoptosis.

CTRP6 inhibits RhoA/Rock/PTEN signaling and activates the PI3K/Akt pathway. To explore whether RhoA and Akt-associated signaling are the targets of CTRP6, western blotting was used to detect the protein expression of GTP-RhoA, Rock1, PTEN, phosphorylated (p)-PI3K and p-Akt. The data revealed enhanced expression of GTP-RhoA, Rock1 and PTEN, and reduced p-PI3K and p-Akt levels upon OGD/R treatment (Fig. 5), indicating that the RhoA/Rock/PTEN and PI3K/Akt pathways are involved in cerebral I/R injury. Next, CTRP6 overexpression was used to determine its effect on RhoA/Rock/PTEN and PI3K/Akt signaling, and it was observed that CTRP6 overexpression inhibited RhoA, Rock and PTEN expression, but reactivated PI3K and Akt expression in a cerebral I/R injury cell model (Fig. 5).

Overexpression of RhoA abolishes, while the PTEN inhibitor recovers, the protective effects of CTRP6 against $O G D / R$-induced inflammation, oxidative stress and cell apoptosis. To further confirm the role of the RhoA/Rock/PTEN and PI3K/Akt pathways in the protective effects of CTRP6 against cerebral I/R injury, recombinant plasmids expressing RhoA were transfected into PC12 cells to overexpress RhoA 

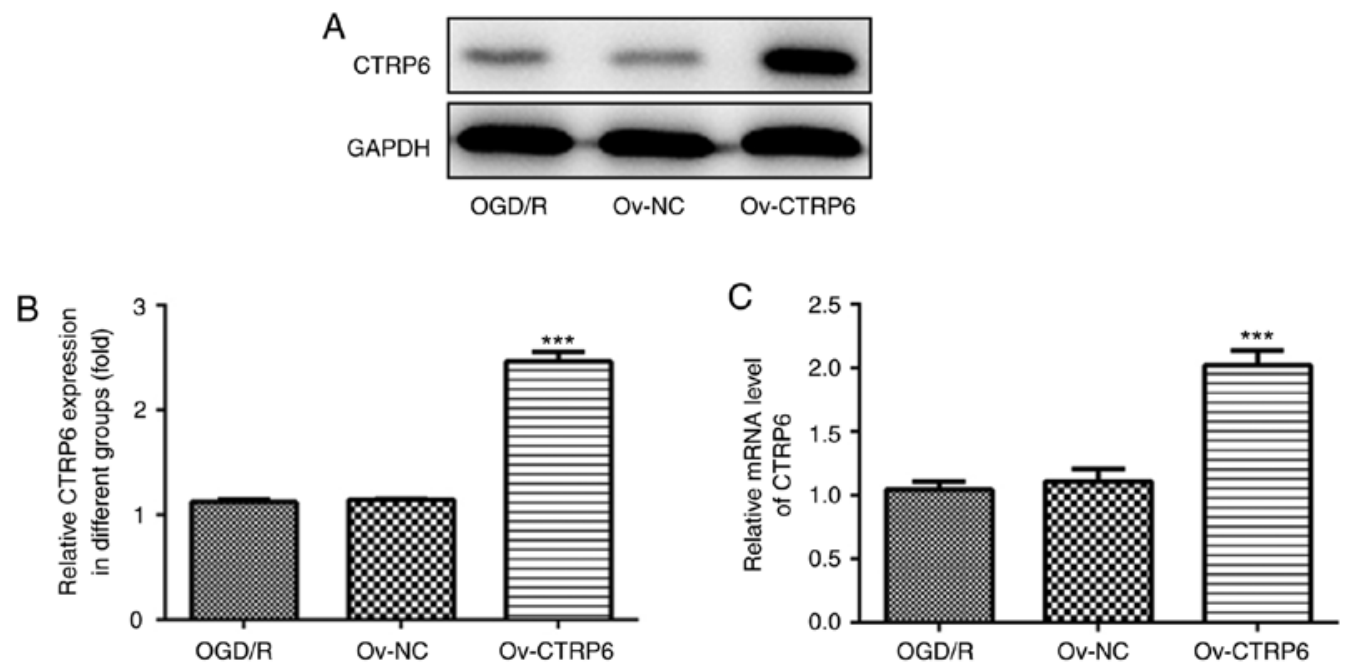

Figure 2. Overexpression of CTRP6 in PC12 cells. (A) Representative immunoblot analysis for CTRP6 in PC12 cells. (B) Relative CTRP6 protein expression normalized to the control $(n=3)$. (C) Relative mRNA level of CTRP6 $(n=3) .{ }^{* * * *} \mathrm{P}<0.001$ vs. Ov-NC. CTRP6, C1q/tumor necrosis factor-related protein-6; Ov, overexpression; NC, negative control.
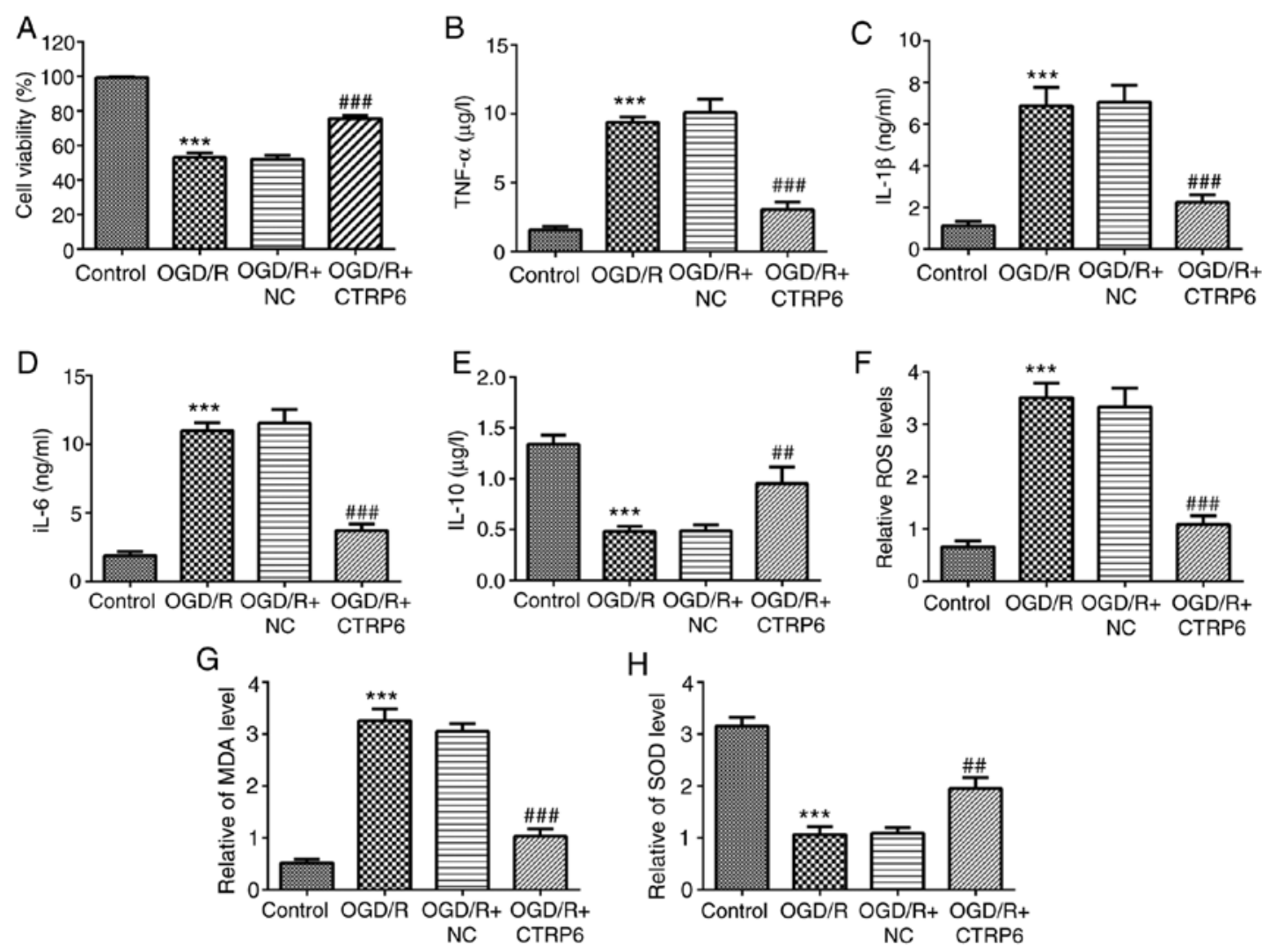

Figure 3. CTRP6 overexpression inhibits OGD/R-induced inflammation and oxidative stress in PC12 cells. Alteration of (A) cell viability and inflammatory cytokines (B) TNF- $\alpha,(C)$ IL-1 $\beta$, (D) IL-6 and (E) IL-10 levels (n=3). Alteration of oxidative products (F) ROS, (G) MDA and (H) antioxidant SOD levels (n=3). ${ }^{* * *} \mathrm{P}<0.001$ vs. control, ${ }^{\# \#} \mathrm{P}<0.01$ and ${ }^{\# \# /} \mathrm{P}<0.001$ vs. OGD/R + NC. CTRP6, C1q/tumor necrosis factor-related protein-6; OGD/R, oxygen-glucose deprivation and reperfusion; NC, negative control; TNF, tumor necrosis factor; IL, interleukin; ROS, reactive oxygen species; MDA, malondialdehyde; SOD, superoxide dismutase.

(Fig. 6A-C). The results of western blotting demonstrated that the CTRP6-stimulated activation of the PI3K/Akt pathway was blunted by RhoA overexpression (Fig. 6D-F). However, the expression of PI3K and the level of p-Akt were rescued when the cells were treated with a PTEN inhibitor (Fig. 6D-F). Consequently, it was confirmed that CTRP6 activates the
PI3K/Akt pathway via inhibiting RhoA/Rock/PTEN signaling in OGD/R-induced PC12 cells.

To elucidate the direct effect of RhoA and PTEN on CTRP6 against OGD/R-induced cerebral I/R injury, the levels of cytokines and proteins associated with inflammation, oxidative stress and apoptosis were detected when cells overexpressed 

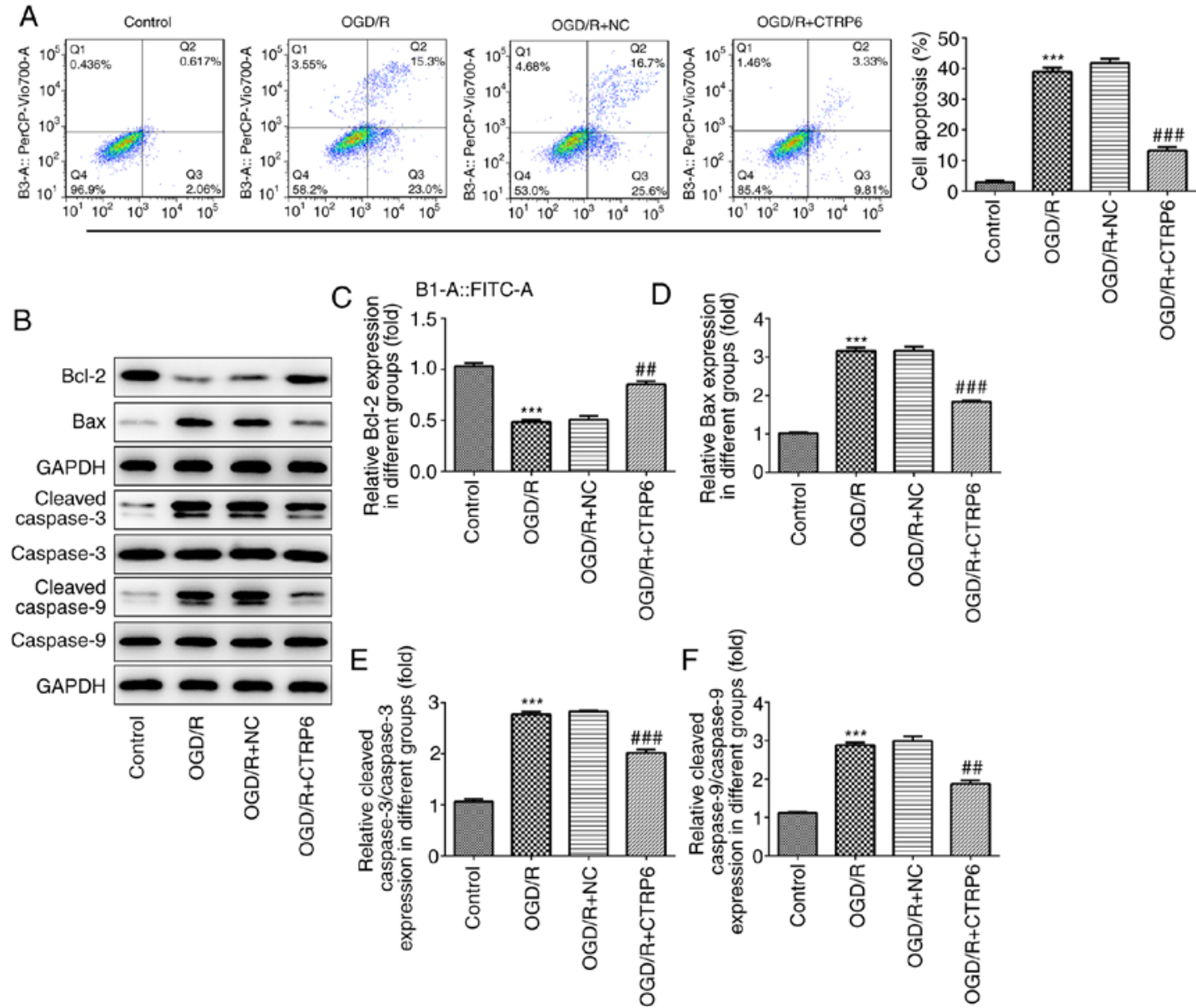

Figure 4. CTRP6 overexpression prevents OGD/R-induced apoptosis in PC12 cells. (A) Cell apoptosis was assessed by flow cytometry and the ratio of apoptotic cells in each group was calculated. (B) Representative immunoblot analysis for apoptosis-related proteins in PC12 cells. Relative protein expression of (C) anti-apoptosis protein Bcl-2 and pro-apoptosis proteins (D) Bax, (E) cleaved caspase-3 and (F) cleaved caspase-9 following normalization to GAPDH $(\mathrm{n}=3) .{ }^{* * *} \mathrm{P}<0.001$ vs. control, ${ }^{\# \#} \mathrm{P}<0.01$ and ${ }^{\# \# \#} \mathrm{P}<0.001$ vs. OGD/R + NC. CTRP6, C1q/tumor necrosis factor-related protein-6; OGD/R, oxygen-glucose deprivation and reperfusion; $\mathrm{NC}$, negative control.
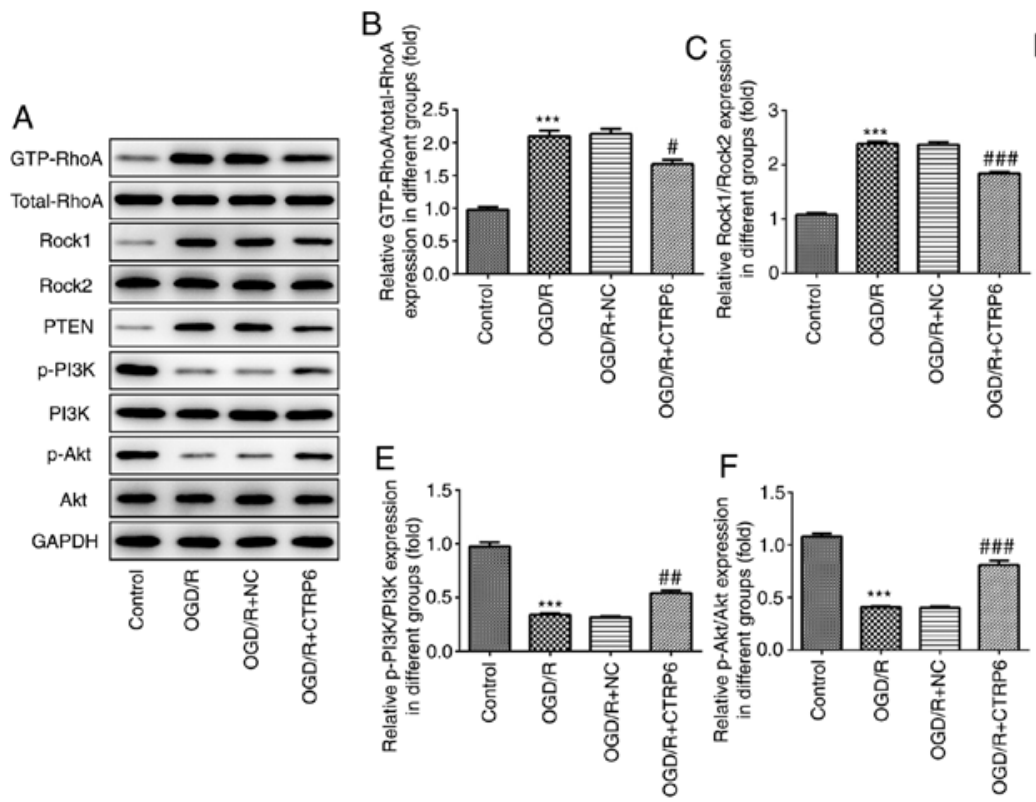

Figure 5. CTRP6 overexpression inhibits RhoA/Rock/PTEN and activates the PI3K/Akt signaling pathway. (A) Representative immunoblot analysis for RhoA, Rock1, PTEN, p-PI3K and p-Akt. Relative protein expression of (B) RhoA, (C) Rock1, (D) PTEN, (E) p-PI3K and (F) p-Akt after normalization to GAPDH $(\mathrm{n}=3) .{ }^{* * *} \mathrm{P}<0.001$ vs. control, ${ }^{\#} \mathrm{P}<0.05,{ }^{\# \#} \mathrm{P}<0.01$ and ${ }^{\# \# /} \mathrm{P}<0.001$ vs. OGD/R + NC. CTRP6, Clq/tumor necrosis factor-related protein-6; OGD/R, oxygen-glucose deprivation and reperfusion; NC, negative control; RhoA, Ras homolog family member A; Rock, Rho-associated coiled-coil-containing protein kinase; PTEN, phosphatase and tensin homologue deleted on chromosome 10; PI3K, phosphoinositide 3-kinase; Akt, protein kinase B; p, phosphorylated. 

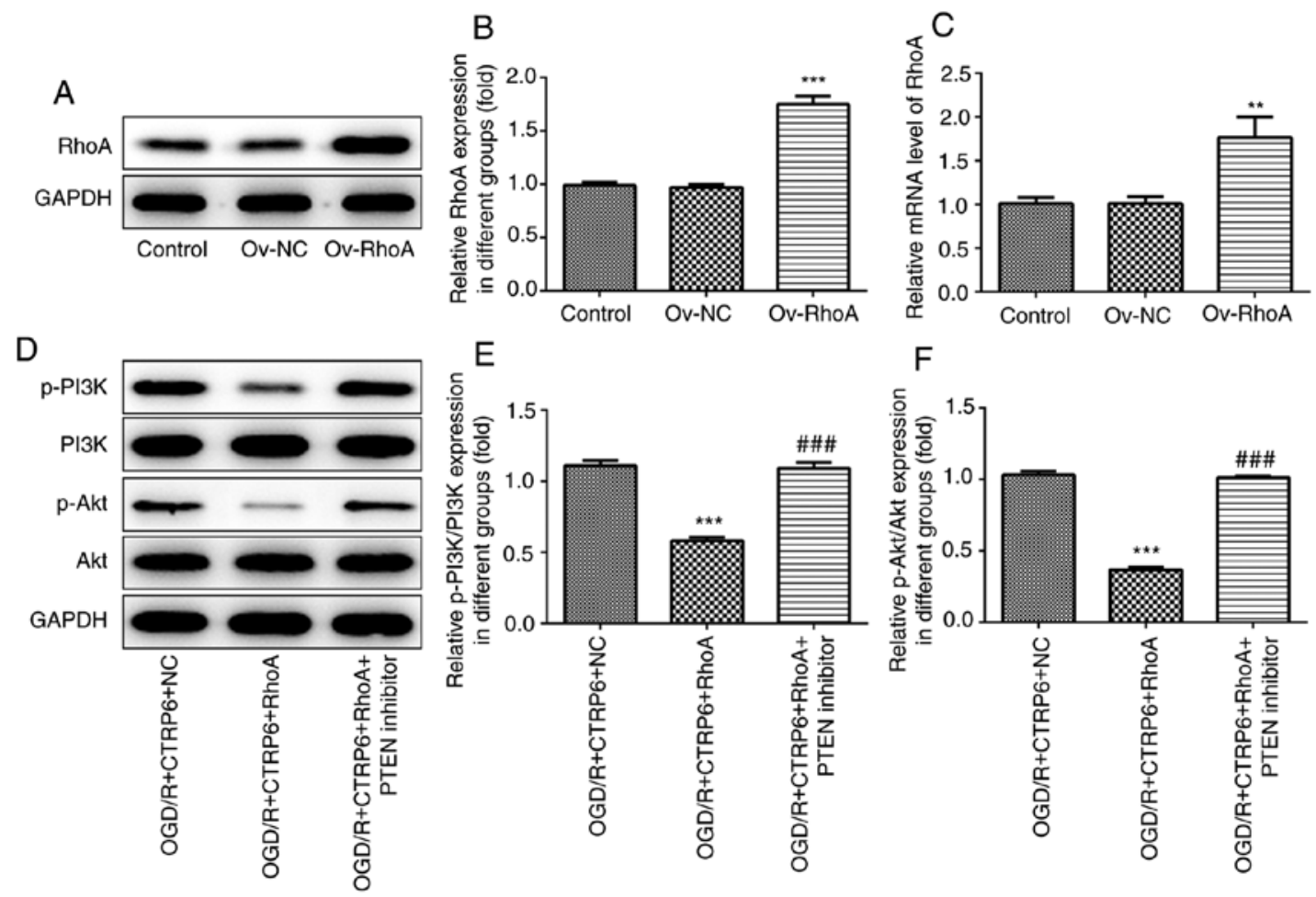

Figure 6. RhoA overexpression attenuates, while PTEN inhibitor rescues the activation of PI3K and Akt induced by CTRP6. (A) Overexpression of RhoA was verified by western blotting. (B and C) Relative protein and mRNA expression of RhoA in different groups ( $\mathrm{n}=3$ ). ${ }^{* * *} \mathrm{P}<0.01$ and ${ }^{* * * *} \mathrm{P}<0.001 \mathrm{vs}$. ov-NC. (D) Representative immunoblot analysis for p-PI3K and p-Akt. Relative protein expression of (E) p-PI3K and (F) p-Akt after normalization to GAPDH (n=3). ${ }^{* * *} \mathrm{P}<0.001$ vs. OGD/R + CTRP6 + NC, ${ }^{\# \#} \mathrm{P}<0.001$ vs. OGD/R + CTRP6 + RhoA. CTRP6, C1q/tumor necrosis factor-related protein-6; OGD/R, oxygen-glucose deprivation and reperfusion; NC, negative control; RhoA, Ras homolog family member A; PTEN, phosphatase and tensin homologue deleted on chromosome 10; PI3K, phosphoinositide 3-kinase; p-Akt, phosphorylated-protein kinase B.

A

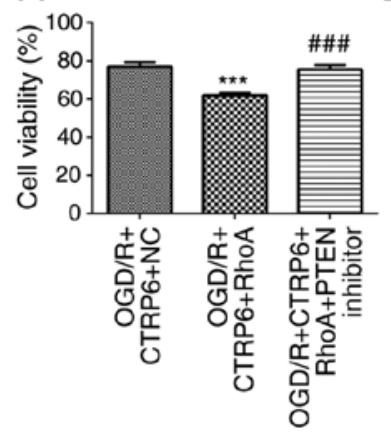

E

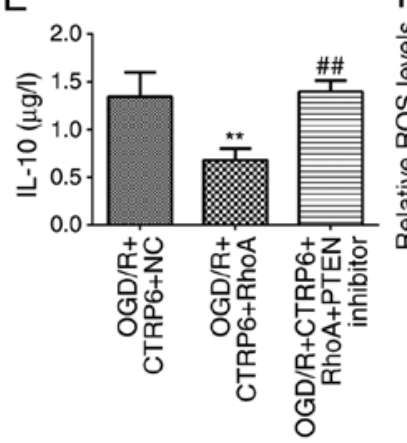

B

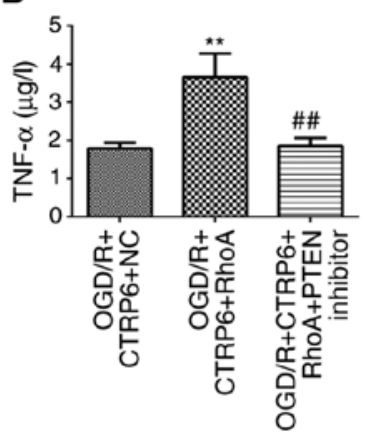

$\mathrm{F}$

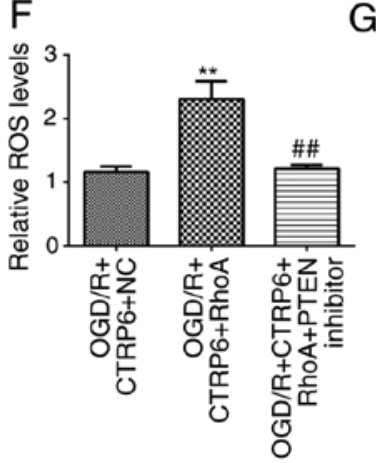

C

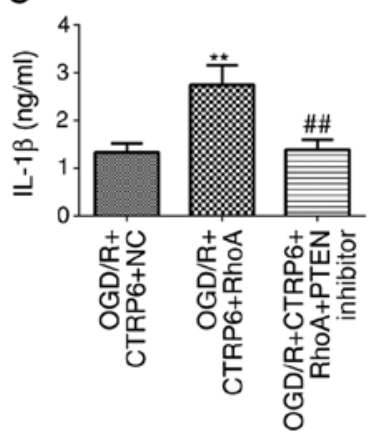

D
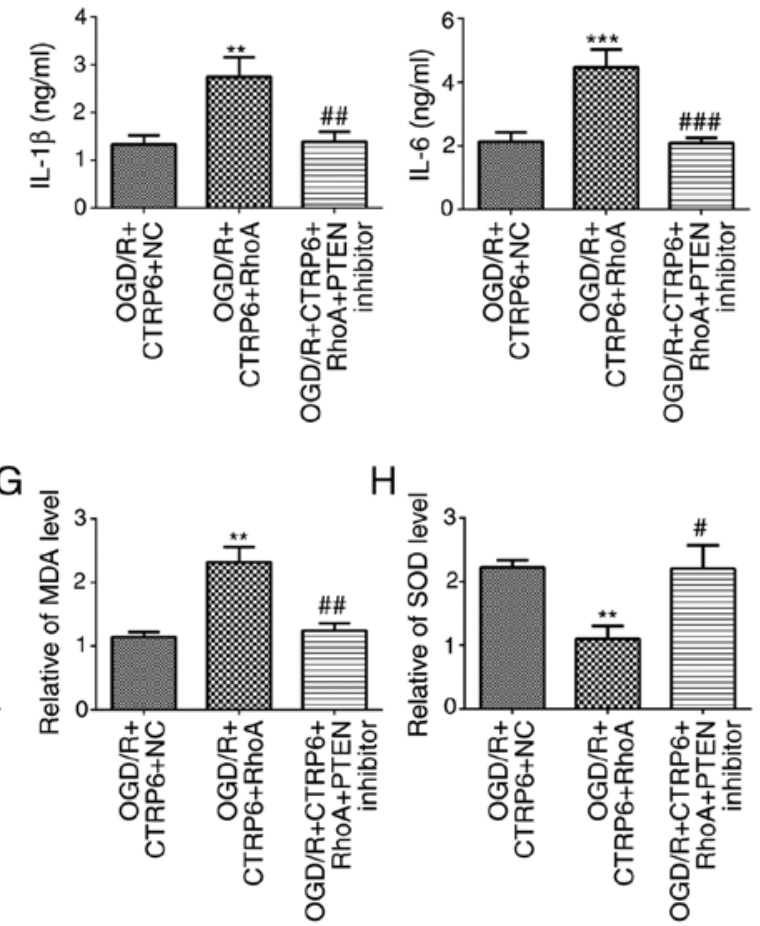

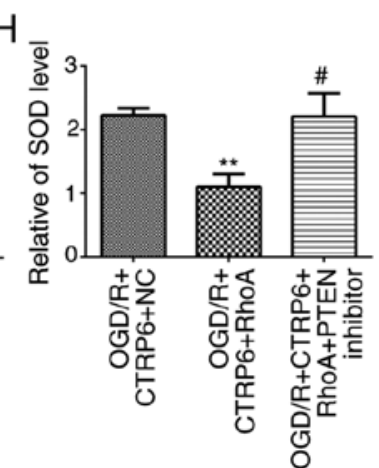

Figure 7. RhoA overexpression abolishes, while the PTEN inhibitor recovers, the protective effects of CTRP6 against OGD/R-induced inflammation and oxidative stress. Alteration of (A) cell viability and inflammatory cytokines (B) TNF- $\alpha$, (C) IL-1 $\beta$, (D) IL-6 and (E) IL-10 levels in different groups ( $\mathrm{n}=3$ ). Alteration of oxidative products $(\mathrm{F}) \mathrm{ROS},(\mathrm{G}) \mathrm{MDA}$ and $(\mathrm{H})$ antioxidant SOD levels in different groups $(\mathrm{n}=3){ }^{* * *} \mathrm{P}<0.01$ and ${ }^{* * * *} \mathrm{P}<0.001$ vs. OGD/R $+\mathrm{CTRP} 6+$

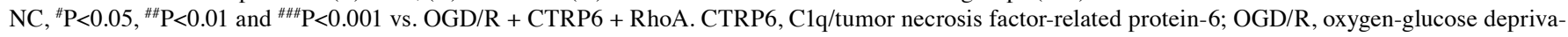
tion and reperfusion; NC, negative control; RhoA, Ras homolog family member A; PTEN, phosphatase and tensin homologue deleted on chromosome 10; TNF, tumor necrosis factor; IL, interleukin; ROS, reactive oxygen species; MDA, malondialdehyde; SOD, superoxide dismutase. 

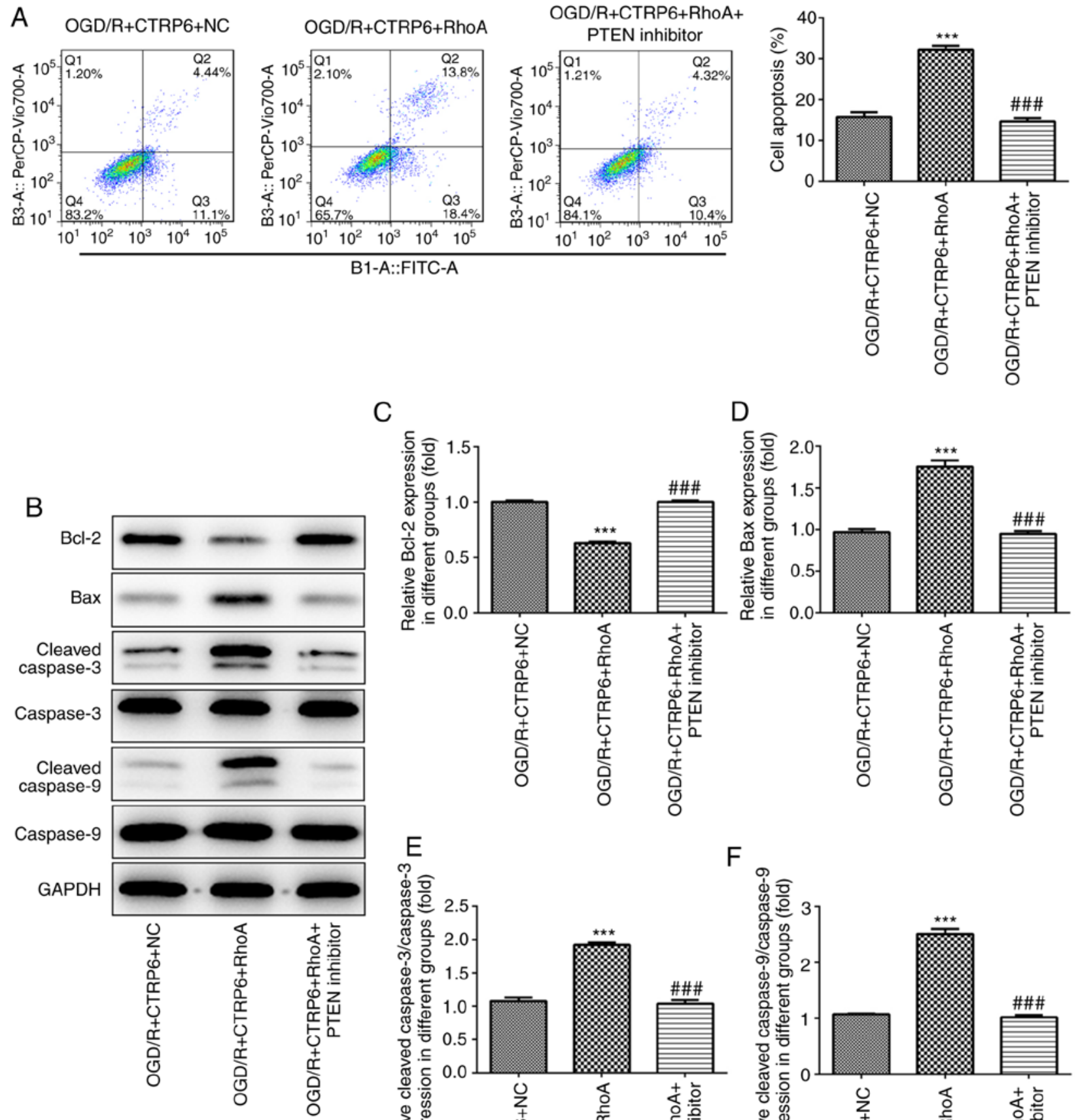

C
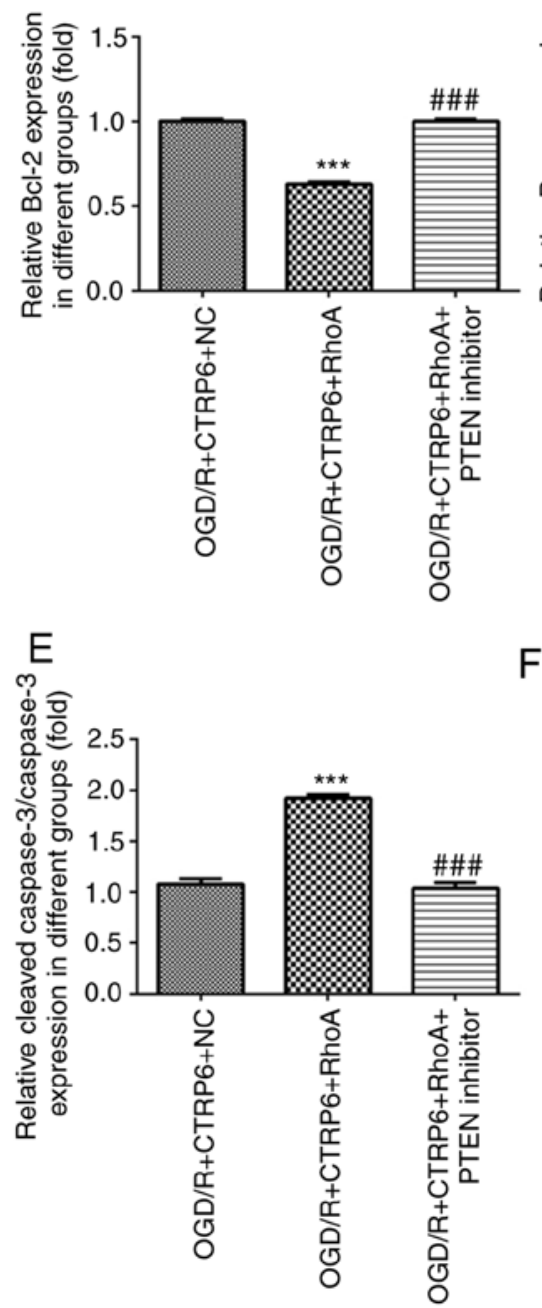

D

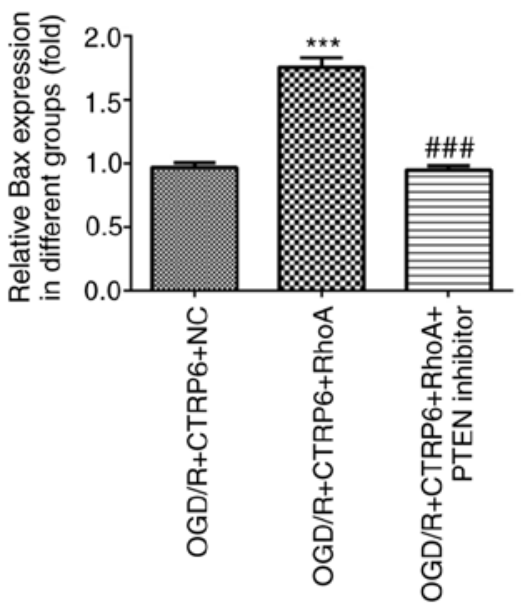

$\mathrm{F}$

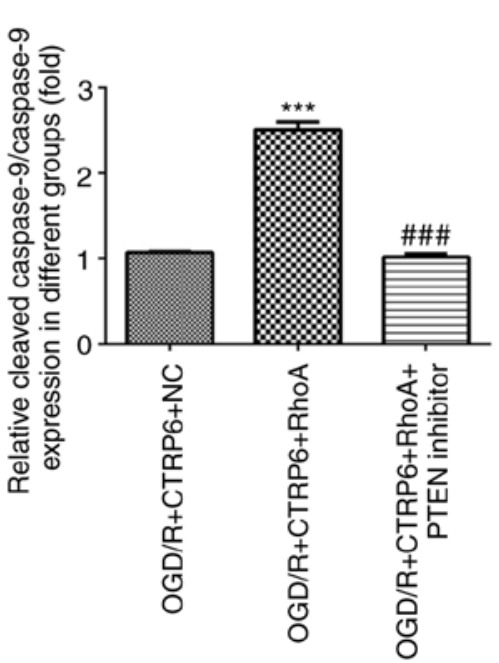

Figure 8. RhoA overexpression abolishes, while the PTEN inhibitor rescues, the protective effects of CTRP6 against OGD/R-induced apoptosis. (A) Cell apoptosis was assessed by flow cytometry and the ratio of apoptotic cells in each group was calculated. (B) Representative immunoblot analysis for apoptosis-related proteins. Relative protein expression of (C) anti-apoptosis protein Bcl-2 and pro-apoptosis proteins (D) Bax, (E) cleaved caspase-3 and (F) cleaved caspase-9 $(\mathrm{n}=3){ }^{* * * *} \mathrm{P}<0.001$ vs. OGD/R + CTRP6 + NC, ${ }^{\# \# \#} \mathrm{P}<0.001$ vs. OGD/R + CTRP6 + RhoA. CTRP6, C1q/tumor necrosis factor-related protein-6; OGD/R, oxygen-glucose deprivation and reperfusion; NC, negative control; RhoA, Ras homolog family member A; PTEN, phosphatase and tensin homologue deleted on chromosome 10; FITC, fluorescein isothiocyanate.

RhoA or were treated with a PTEN inhibitor. First, an ELISA was applied to evaluate the activities of inflammation-related factors. As seen from the data, the CTRP6-stimulated decrease in the levels of TNF- $\alpha$, IL-1 $\beta$, IL- 6 and increase in the levels of IL-10 was diminished by RhoA overexpression, whereas it was rescued by the PTEN inhibitor (Figs. 3B-E and 7B-E). In the same manner, RhoA overexpression cancelled, while PTEN inhibitor rescued the alleviation of oxidative stress caused by CTRP6 (Figs. 3F-H and 7F-H). Finally, the ratio of apoptotic cells and apoptosis-related protein expression were also 
evaluated by flow cytometry and western blotting. RhoA overexpression and PTEN inhibitor exerted the same effect on the protective action of CTRP6 against apoptosis (Figs. 4 and 8).

\section{Discussion}

To the best of our knowledge, the present study is the first to document the pivotal role of CTRP6 in regulating RhoA/Rock/PTEN and PI3K/Akt signaling-mediated inflammation, oxidative stress and apoptosis in I/R injury. Consistent with previously reported studies $(6,8)$, the present results verified the participation of inflammation, oxidative stress and apoptosis in I/R injury. Due to the significant decrease of CTRP6 level in OGD/R-treated PC12 cells, the replenishment of CTRP6 may be effective in reducing cerebral I/R damage. In accordance with the current hypothesis, overexpression of CTRP6 before the induction of OGD/R led to a reduction in inflammation, oxidative stress and apoptosis following I/R injury in PC12 cells.

A recent report demonstrated that CTRP6 protected cardiomyocytes from doxorubicin-induced apoptosis and activated the Akt pathway in vitro (17). In the present study, CTRP6 overexpression enhanced activation of Akt and its upstream regulator PI3K, and inhibited the activation of RhoA, Rock and PTEN in OGD/R-treated PC12 cells. Rock is a major downstream effector of RhoA, while PTEN has been identified as a Rock substrate as well as a negative regulator of the PI3K/Akt pathway, which plays important roles in cell survival and apoptosis (25-27). In previous years, an increasing number of studies have confirmed that the RhoA/Rock/PTEN/PI3K/Akt signaling network also plays a crucial role in $\mathrm{I} / \mathrm{R}$ injury $(21-23,28)$. Therefore, it was inferred that replenishment of CTRP6 could protect against OGD/R-induced injury via inhibiting the RhoA/Rock/PTEN signaling pathway, thereby activating PI3K/Akt signaling.

To validate the present hypothesis, RhoA was overexpressed, with or without treatment with the PTEN inhibitor, to observe the effect of CTRP6 on OGD/R-induced injury. As expected, the protective effects of CTRP6 against OGD/R-induced inflammation, oxidative stress and cell apoptosis were all diminished by RhoA overexpression; however, the presence of the PTEN inhibitor recovered the protective effects of CTRP6.

As an adipocytokine similar to other members of the CTRP family, CTRP6 was shown to play a key role in obesity, diabetes and vascular diseases (29-31); however, the role of CTRP6, as well as that of other CTRPs, in cerebral I/R injury remains elusive. Previous studies have suggested the protective effects of CTRP1 and CTRP9 against ischemia in the heart $(20,32)$, but the specific role of CTRPs in ischemic diseases is poorly understood. The current study provided evidence that CTRP6 exerts neuroprotective effects against I/R injury through the RhoA/Rock/PTEN/PI3K/Akt signaling network. Further in vitro and in vivo research is required to investigate the functions of CTRP6 and other CTRP members in cerebral I/R injury.

In conclusion, the present study demonstrated that CTRP6 acts as a novel regulator of cerebral I/R-induced inflammation, oxidative stress and cell apoptosis through inhibition of the RhoA/Rock/PTEN pathway and activation of PI3K/Akt signaling. Therefore, enhancing CTRP6 production may be of value for the prevention and/or treatment of cerebral I/R injury.

\section{Acknowledgements}

Not applicable.

\section{Funding}

The present study was supported by a grant from the Key Medical Discipline Development Programs of Beijing Hospitals Authority (grant no. ZYLX201832).

\section{Availability of data and materials}

All data generated or analyzed during this study are included in this published article.

\section{Authors' contributions}

XG and YL conceived and designed the study; YL and JS performed the experiments and acquired the data; LG analyzed and interpreted the data; and YL and XG drafted the manuscript and revised it critically for important intellectual content. All authors read and approved the final manuscript.

\section{Ethics approval and consent to participate}

Not applicable.

\section{Patient consent for publication}

Not applicable.

\section{Competing interests}

The authors declare that they have no competing interests.

\section{References}

1. Prabhakaran S, Ruff I and Bernstein RA: Acute stroke intervention: A systematic review. JAMA 313: 1451-1462, 2015.

2. Palomares SM and Cipolla MJ: Vascular protection following cerebral ischemia and reperfusion. J Neurol Neurophysiol 2011: pii: S1-004, 2011.

3. Thompson BJ and Ronaldson PT: Drug delivery to the ischemic brain. Adv Pharmacol 71: 165-202, 2014.

4. Sanderson TH, Reynolds CA, Kumar R, Przyklenk K and Huttemann M: Molecular mechanisms of ischemia-reperfusion injury in brain: Pivotal role of the mitochondrial membrane potential in reactive oxygen species generation. Mol Neurobiol 47: 9-23, 2013.

5. Gong L, Tang Y, An R, Lin M, Chen L and Du J: RTN1-C mediates cerebral ischemia/reperfusion injury via ER stress and mitochondria-associated apoptosis pathways. Cell Death Dis 8: e3080, 2017.

6. Zhang DD, Zou MJ, Zhang YT, Fu WL, Xu T, Wang JX, Xia WR, Huang ZG, Gan XD, Zhu XM and Xu DG: A novel IL-1RA-PEP fusion protein with enhanced brain penetration ameliorates cerebral ischemia-reperfusion injury by inhibition of oxidative stress and neuroinflammation. Exp Neurol 297: 1-13, 2017.

7. Wu H, Tang C, Tai LW, Yao W, Guo P, Hong J, Yang X, Li X, Jin Z, Ke J and Wang Y: Flurbiprofen axetil attenuates cerebral ischemia/reperfusion injury by reducing inflammation in a rat model of transient global cerebral ischemia/reperfusion. Biosci Rep 38: pii: BSR20171562, 2018. 
8. Majid A: Neuroprotection in stroke: Past, present, and future. ISRN Neurol 2014: 515716, 2014.

9. Yu ZH, Cai M, Xiang J, Zhang ZN, Zhang JS, Song XL, Zhang W, Bao J, Li WW and Cai DF: PI3K/Akt pathway contributes to neuroprotective effect of Tongxinluo against focal cerebral ischemia and reperfusion injury in rats. J Ethnopharmacol 181: 8-19, 2016.

10. Zhang Q, An R, Tian X, Yang M, Li M, Lou J, Xu L and Dong Z: $\beta$-Caryophyllene pretreatment alleviates focal cerebral ischemia-reperfusion injury by activating PI3K/Akt signaling pathway. Neurochem Res 42: 1459-1469, 2017.

11. Zhang JF, Zhang L, Shi LL, Zhao ZH, Xu H, Liang F, Li HB, Zhao Y, Xu X, Yang K and Tian YF: Parthenolide attenuates cerebral ischemia/reperfusion injury via Akt/GSK-3 $\beta$ pathway in PC12 cells. Biomed Pharmacother 89: 1159-1165, 2017.

12. Schaffler A and Buechler C: CTRP family: Linking immunity to metabolism. Trends Endocrinol Metab 23: 194-204, 2012.

13. Wong GW,Krawczyk SA, Kitidis-Mitrokostas C,Revett T, Gimeno R and Lodish HF: Molecular, biochemical and functional characterizations of C1q/TNF family members: Adipose-tissue-selective expression patterns, regulation by PPAR-gamma agonist, cysteine-mediated oligomerizations, combinatorial associations and metabolic functions. Biochem J 416: 161-177, 2008.

14. Ohashi K, Shibata R, Murohara T and Ouchi N: Role of anti-inflammatory adipokines in obesity-related diseases. Trends Endocrinol Metab 25: 348-355, 2014.

15. Kim MJ,Lee W, Park EJ and Park SY: ClqTNF-related protein-6 increases the expression of interleukin-10 in macrophages. Mol Cells 30: 59-64, 2010.

16. Lei H, Wu D, Wang JY, Li L, Zhang CL, Feng H, Fu FY and Wu LL: $\mathrm{Clq} /$ tumor necrosis factor-related protein- 6 attenuates post-infarct cardiac fibrosis by targeting RhoA/MRTF-A pathway and inhibiting myofibroblast differentiation. Basic Res Cardiol 110: 35, 2015.

17. Zheng WF, Zhang SY, Ma HF, Chang XW and Wang $\mathrm{H}$ : ClqTNF-related protein- 6 protects against doxorubicin-induced cardiac injury. J Cell Biochem 120: 10748-10755, 2019.

18. Murayama MA, Kakuta S, Inoue A, Umeda N, Yonezawa T, Maruhashi T, Tateishi K, Ishigame $\mathrm{H}$, Yabe R, Ikeda $\mathrm{S}$, et al CTRP6 is an endogenous complement regulator that can effectively treat induced arthritis. Nat Commun 6: 8483, 2015.

19. Zhao D, Feng P, Sun Y, Qin Z, Zhang Z, Tan Y, Gao E, Lau WB, Ma X, Yang J, et al: Cardiac-derived CTRP9 protects against myocardial ischemia/reperfusion injury via calreticulin-dependent inhibition of apoptosis. Cell Death Dis 9: 723, 2018.

20. Kambara T,Ohashi K, ShibataR, Ogura Y,MaruyamaS, Enomoto T, Uemura Y, Shimizu Y, Yuasa D, Matsuo K, et al: CTRP9 protein protects against myocardial injury following ischemia-reperfusion through AMP-activated protein kinase (AMPK)-dependent mechanism. J Biol Chem 287: 18965-18973, 2012.

21. Zhong Y, Yu C and Qin W: LncRNA SNHG14 promotes inflammatory response induced by cerebral ischemia/reperfusion injury through regulating miR-136-5p /ROCK1. Cancer Gene Ther 26: 234-247, 2019.
22. Lu L, Yue S, Jiang L, Li C, Zhu Q, Ke M, Lu H, Wang X, Busuttil RW, Ying QL, et al: Myeloid Notch1 deficiency activates the RhoA/ROCK pathway and aggravates hepatocellular damage in mouse ischemic livers. Hepatology 67: 1041-1055, 2018.

23. Chang J, Yao X, Zou H, Wang L, Lu Y, Zhang Q and Zhao H: BDNF/PI3K/Akt and Nogo-A/RhoA/ROCK signaling pathways contribute to neurorestorative effect of Houshiheisan against cerebral ischemia injury in rats. J Ethnopharmacol 194: 1032-1042, 2016.

24. Livak KJ and Schmittgen TD: Analysis of relative gene expression data using real-time quantitative PCR and the 2(-Delta Delta C(T)) method. Methods 25: 402-408, 2001.

25. Yang S and Kim HM: The RhoA-ROCK-PTEN pathway as a molecular switch for anchorage dependent cell behavior. Biomaterials 33: 2902-2915, 2012.

26. Li G, Liu L, Shan C, Cheng Q, Budhraja A, Zhou T, Cui H and Gao N: RhoA/ROCK/PTEN signaling is involved in AT-101-mediated apoptosis in human leukemia cells in vitro and in vivo. Cell Death Dis 5: e998, 2014.

27. Papakonstanti EA, Ridley AJ and Vanhaesebroeck B: The p110delta isoform of PI 3-kinase negatively controls RhoA and PTEN. EMBO J 26: 3050-3061, 2007.

28. Lauriol J, Keith K, Jaffre F, Couvillon A, Saci A, Goonasekera SA, McCarthy JR, Kessinger CW, Wang J, Ke Q, et al: RhoA signaling in cardiomyocytes protects against stress-induced heart failure but facilitates cardiac fibrosis. Sci Signal 7: ra100, 2014.

29. Lei X, Seldin MM, Little HC, Choy N, Klonisch T and Wong GW: C1q/TNF-related protein 6 (CTRP6) links obesity to adipose tissue inflammation and insulin resistance. J Biol Chem 292 14836-14850, 2017.

30. Wang M, Tang X, Li L, Liu D, Liu H, Zheng H, Deng W, Zhao X and Yang G: C1q/TNF-related protein- 6 is associated with insulin resistance and the development of diabetes in Chinese population. Acta Diabetol 55: 1221-1229, 2018.

31. Chi L, Hu X, Zhang W, Bai T, Zhang L, Zeng H, Guo R, Zhang Y and Tian H: Adipokine CTRP6 improves PPARY activation to alleviate angiotensin II-induced hypertension and vascular endothelial dysfunction in spontaneously hypertensive rats. Biochem Biophys Res Commun 482: 727-734, 2017.

32. Yuasa D, Ohashi K, Shibata R, Mizutani N, Kataoka Y,Kambara T, Uemura Y, Matsuo K, Kanemura N, Hayakawa S, et al: $\mathrm{C} 1 \mathrm{q} / \mathrm{TNF}-$ related protein-1 functions to protect against acute ischemic injury in the heart. FASEB J 30: 1065-1075, 2016.

(i) $(-)$ This work is licensed under a Creative Commons Attribution-NonCommercial-NoDerivatives 4.0 International (CC BY-NC-ND 4.0) License. 